\title{
Aplicativo para smartphone como estratégia de ensino das manobras de higiene brônquica para fisioterapia
}

\author{
Smartphone application as a teaching strategy for bronchy \\ hygiene manuals for physiotherapy
}

\author{
Renato Caldas dos Santos ${ }^{1}$, Patrick Abdala Fonseca Gomes ${ }^{2}$ \\ ${ }^{1}$ Autor para correspondência. Centro Universitário do Pará. Belém, Pará, Brasil. ORCID: 0000-0002-1029-3965. recal@superig.com.br \\ ${ }^{2}$ Centro Universitário do Pará. Belém, Pará, Brasil. ORCID: 0000-0001-7577-7736. abdalagomes@me.com
}

\begin{abstract}
RESUMO | INTRODUÇÃO: No decorrer dos anos, os cursos da saúde têm passado por importantes mudanças na velocidade das inovações, trazendo o crescimento, pesquisa e melhoria para o processo da educação. A introdução de tecnologias e novos métodos de ensino têm contribuído para aprimorar e permitir o aprendizado de maneira mais eficiente. OBJETIVO: desenvolver e aplicar vídeos de manobras de higiene brônquica, através de aplicativo, como ferramenta de ensino para graduandos de fisioterapia do CESUPA. MATERIAIS E MÉTODOS: $O$ estudo realizado foi experimental e descritivo. Foram analisados 40 alunos do estágio supervisionado de fisioterapia, dos $7^{\circ}$ e $8^{\circ}$ períodos de 2018, também foram analisados 4 juízes com comprovação na docência em curso de fisioterapia. Foi criado um aplicativo móvel, com vídeos de manobras de higiene brônquica, foi feita a validação do aplicativo móvel pelos juízes e, por fim, verificou-se a opinião dos alunos quanto à utilização do aplicativo móvel proposto para o processo de ensino-aprendizagem. RESULTADO: Referentes à atenção, relevância, confiança, conhecimento, compreensão e aplicação, a avaliação foi satisfatória, totalizando $60 \%$ dos para graduandos de fisioterapia. CONCLUSÃO: Após a análise das respostas, concluirmos, em sua totalidade, a satisfação dos juízes, salientando que o aplicativo é prático, de fácil entendimento e pode auxiliar na aprendizagem. Na avalição das opiniões geradas pelos alunos, quanto a utilização do aplicativo móvel proposto para o processo de ensino-aprendizagem, nos aspectos, instrumento de aprendizagem, ferramenta de estudo, facilidade de manuseio, na promoção de dados concretos para o ensino das manobras de higiene brônquica, auxiliar na prática da fisioterapia, a maioria significativa dos alunos considerou o aplicativo avaliado como excelente.
\end{abstract}

PALAVRAS-CHAVE: Aplicativos móveis. Manobra. Ensino. Aprendizagem. Recursos audiovisuais.
ABSTRACT | INTRODUCTION: Over the years, health courses have undergone important changes in the speed of innovation, bringing growth, research and improvement to the education process. The introduction of technologies and new teaching methods have contributed to improve and enable learning more efficiently. OBJECTIVE: To develop and apply videos of bronchial hygiene maneuvers, through application, as a teaching tool for physiotherapy students. MATERIALS AND METHODS: The study was experimental and descriptive. Forty students from the supervised physiotherapy internship, from the 7th and 8th periods of 2018, were analyzed. Four judges were also verified with the teaching in physiotherapy course. A mobile app was created, with videos of bronchial hygiene maneuvers, the mobile app was validated by the judges and, finally, the students' opinion regarding the use of the proposed mobile app for the teachinglearning process was verified. RESULT: Regarding attention, relevance, confidence, knowledge, understanding and application, the assessment was satisfactory, totaling $60 \%$ of those for physiotherapy students. CONCLUSION: After analyzing the answers, we fully concluded the satisfaction of the judges, noting that the application is practical, easy to understand and can help learning. In the evaluation of the opinions generated by the students, regarding the use of the proposed mobile application for the teaching-learning process, in the aspects, learning instrument, study tool, ease of handling, promoting concrete data for teaching hygiene maneuvers. bronchial aids, assisting in the practice of physical therapy, the significant majority of students considered the evaluated application as excellent.

KEYWORDS: Mobile applications. Maneuver. Teaching. Learning. audiovisual Aids. 


\section{Introdução}

O ensino em saúde encontra-se sob crítica recorrente em todo o mundo. No Brasil, tornou-se objeto de análise pelos profissionais da área e pela sociedade em geral. Existe, de certa forma, um consenso quanto à necessidade de reformulação de determinados aspectos da formação com vistas a uma satisfação das demandas assistenciais atuais' ${ }^{1}$.

As atuais políticas nacionais de saúde e de educação apontam para a necessidade de mudanças nos processos de formação profissional em saúde, tem estimulo e apoio para ampliar a responsabilidade social e a pactuação de propostas para definição de competências e para o desenvolvimento das diretrizes curriculares nacionais ${ }^{2}$.

Essas mudanças paradigmáticas, envolvendo as concepções de saúde e educação, precisam ser vivenciadas durante a formação, ampliando as possibilidades de horizontalização e democratização do conhecimento. Competências e habilidades também precisam ser desenvolvidas pelos profissionais de saúde, para que se constituam como sujeitos comprometidos com a busca da apreciação do cuidado².

No decorrer dos anos, os cursos de saúde têm passado por importantes mudanças na velocidade das inovações, trazendo o crescimento, pesquisa e melhoria para o processo da educação. A introdução de tecnologias e novos métodos de ensino, tem contribuído para aprimorar e permitir o aprendizado de maneira mais precoce. Os recursos tecnológicos de comunicação e informação tem se desenvolvido de forma rápida, ocupando espaço no cotidiano de alunos e professores, servindo como ferramenta de auxilio no processo ensino e aprendizagem ${ }^{3,4}$. Esses reflexos da "era digital", estão presentes na educação, com a nova realidade, educadores passaram a repensar a forma tradicional de ensino, incorporando a tecnologia em seu trabalho. Falando a "linguagem do aluno", à compreensão do que está sendo estudado, tornando-se fácil e prazeroso $0^{3,5,6}$.

Dentro do novo panorama digital, os professores precisam continuar no monitoramento do processo de ensino e aprendizagem, atualizando-se e utilizando as novas tecnologias aplicadas nesse processo.
Para isso é preciso que haja, primeiramente, interesse e iniciativa por parte de quem media o conhecimento, aprimorando seus métodos de ensino de acordo com o desenvolvimento tecnológico ${ }^{6}$.

Diante desse cenário tecnológico, a utilização de imagens consiste em importante ferramenta no processo educativo. Nos vídeos, evidencia-se que a qualidade das imagens, podem dar contexto e autenticidade aos ambientes de aprendizagem, fazendo com que a mensagem a ser compartilhada se aproxime ainda mais da realidade. Facilitando ainda, por ser uma ferramenta que permite ao aluno selecionar os conteúdos na qual quer ter conhecimento, podendo avançar, parar, voltar, anotar e estabelecer associação do vídeo com outras fontes de informação ${ }^{4,7,8}$.

A aprendizagem multimídia propõe que as pessoas aprendem melhor quando as imagens são pactuadas com palavras em um ambiente de aprendizagem eletrônica, incluindo a animação e narração, não apenas texto e ilustrações estáticas. Porém, é essencial que as mensagens a transmitir estejam de acordo com o processo cognitivo de cada indivíduo 9 . O uso deste recurso tem um impacto positivo na aprendizagem do aluno em terapia respiratória, campo em que recursos instrumentais e manuais são frequentemente utilizados e podem ser explorado usando esta tecnologia ${ }^{10}$.

A literatura sobre os recursos manuais da fisioterapia respiratória, ou manobras de higiene brônquica, além de escassa, não descreve de maneira satisfatória suas técnicas. Na maioria das vezes, esses recursos são somente citados, talvez por se tratarem de técnicas exclusivamente manuais e exigem muito mais que experiência prática de quem se aplica do que da sua própria fundamentação cientifica ${ }^{11}$, que algumas técnicas de higiene brônquica são mais utilizadas que outras, tanto pela facilidade da aplicação, quanto pela efetividade.

A utilização de ferramentas computacionais na área de saúde está em crescente expansão, pois esse tipo de suporte proporciona aos profissionais de saúde maior precisão e agilidade em seus trabalhos. Desse modo, entende-se que desenvolver soluções computacionais em formato de aplicativo (APP) apresenta um meio eficaz de disponibilizar a ferramenta e atingir o público alvo desejado. O APP é capaz de personalizar e ampliar as funções desses computadores de bolso chamados de smartphones e tablets ${ }^{12}$. 
Com o intuito de auxiliar na formação de profissionais da saúde, esta pesquisa visa como produto final, o desenvolvimento de um aplicativo que forneça os vídeos tutoriais com informações personalizadas para cada técnica de higiene brônquica. Esse APP poderá ser utilizado por estudantes de fisioterapia, profissionais da prática assistencial e no processo de educação continuada.

\section{Materiais e métodos}

O tipo de estudo realizado nesta pesquisa foi experimental e descritivo. A amostra foi composta por 40 alunos, todos do estágio supervisionado de fisioterapia, e 4 Juízes, profissionais fisioterapeutas que atuavam como docentes em cursos de fisioterapia.

Como critérios de inclusão, foram analisados os alunos do estágio supervisionado de fisioterapia, do $7^{\circ}$ e $8^{\circ}$ período de 2018 e juízes com comprovação na docência em curso de fisioterapia, com conhecimento sobre manobras de higiene brônquica. Foram excluídos da pesquisa alunos e juízes que demostraram alguma deficiência audiovisual que os impedisse de assistir os vídeos e que apresentassem dificuldade de compreensão. A coleta de dados foi realizada em dezembro de 2018.

O presente estudo iniciou-se na construção de quatro vídeos referentes às técnicas de higiene brônquica (aceleração de fluxo expiratório - AFE; drenagem autógena - DA; técnica de expiração forçada - TEF; e expiração lenta total com a glote aberta em decúbito infralateral - ELTGOL), todas essas manobras relatadas e avaliadas no consenso de Lyon - 1994/2000.

A elaboração dos vídeos aconteceu nas seguintes etapas: a) revisão da literatura; b) elaboração do roteiro; c) avaliação do roteiro; d) filmagem das técnicas em ambiente simulado, aplicadas em ator que interpretou um paciente; e) edição do vídeo educativo.

Em seguida, foi criado um aplicativo móvel - APP, com os vídeos de manobras de higiene brônquica, em parceria com especialistas de computação, e finalmente foi feita a validação do produto (APP) pelos juízes e, por fim, verificou-se a opinião dos alunos quanto a utilização do aplicativo móvel proposto para o processo de ensino-aprendizagem.

Os dados foram tratados utilizando estatística descritiva, expressos sob a forma de frequências absoluta e relativa e apresentados em tabelas ou figuras. O QuiQuadrado de aderência foi utilizado para comparar a distribuição de frequências observada em cada categoria de respostas esperadas e resultados com $p \leq 0.05$ foram considerados estatisticamente significativos.

\section{Resultados e discussão}

\section{Apresentação do aplicativo móvel}

Desenvolvemos um aplicativo para smartphone abordando manobras de higiene brônquica voltado para o processo de ensino-aprendizagem dos alunos de graduação em fisioterapia. Inicialmente criamos um ícone para facilitar a visualização do aplicativo no smartphone. Produzimos quatro subdivisões do tema central, correspondente aos módulos, indicando as manobras de higiene brônquica.

O modulo 1 representa o vídeo da manobra AFE; O modulo 2 representa o vídeo da manobra Drenagem Autógena; O modulo 3 representa o vídeo da manobra ELTGOL e o modulo 4 representa o vídeo da manobra TEF. Nesse momento, o aplicativo móvel de manobras de higiene brônquica pode ser acessado pelo link: https://3015970.igen.app/ 


\section{Análise do aplicativo pelos juízes}

As tabelas 1 e 2 apresentam os resultados da concordância dos juízes quanto a uma série de 14 afirmações feitas sobre o aplicativo avaliado, as quais avaliaram os parâmetros nível de motivação (afirmativas 1 a 11) e impacto na aprendizagem (conhecimento) (afirmativas 12 a 14).

Tabela 1. Distribuição dos juízes quanto ao nível de concordância com as afirmativas a respeito do nível de motivação feitas sobre o aplicativo avaliado. Belém, 2018

\begin{tabular}{|c|c|c|c|c|c|}
\hline \multirow[b]{2}{*}{ Aspectos e afirmativas } & \multicolumn{5}{|c|}{ Nível de concordância } \\
\hline & $\begin{array}{c}\text { Discordo } \\
\text { fortemente } \\
n(\%)\end{array}$ & $\begin{array}{c}\text { Discordo } \\
n(\%)\end{array}$ & $\begin{array}{c}\text { Neutro } \\
n(\%)\end{array}$ & $\begin{array}{c}\text { Concordo } \\
n(\%)\end{array}$ & $\begin{array}{c}\text { Concordo } \\
\text { fortemente } \\
n(\%)\end{array}$ \\
\hline \multicolumn{6}{|l|}{ Atenção } \\
\hline 1. O design da interface do aplicativo móvel é atraente. & $0(0 \%)$ & $0(0 \%)$ & $2(50 \%)$ & $2(50 \%)$ & $0(0 \%)$ \\
\hline \multicolumn{6}{|l|}{ Relevância } \\
\hline $\begin{array}{l}\text { 2. Ficou claro para mim como o conteúdo do aplicativo móvel está relacionado com coisas que eu já } \\
\text { sabia sobre as manobras de higiene brônquica. }\end{array}$ & $0(0 \%)$ & $0(0 \%)$ & $0(0 \%)$ & $0(0 \%)$ & $4(100 \%)$ \\
\hline $\begin{array}{l}\text { 3. Eu gostei tanto do aplicativo móvel, que gostaria de aprender ainda mais sobre o assunto abordado } \\
\text { por ele. }\end{array}$ & $0(0 \%)$ & $0(0 \%)$ & $0(0 \%)$ & $1(25 \%)$ & $3(75 \%)$ \\
\hline 4. O conteúdo do aplicativo móvel é bastante relevante para o ensino. & $0(0 \%)$ & $0(0 \%)$ & $0(0 \%)$ & $0(0 \%)$ & $4(100 \%)$ \\
\hline $\begin{array}{l}\text { 5. Eu poderia relacionar o conteúdo do aplicativo móvel com coisas que já fiz, ou pensei relacionado ao } \\
\text { ensino discente. }\end{array}$ & $0(0 \%)$ & $0(0 \%)$ & $0(0 \%)$ & $0(0 \%)$ & $4(100 \%)$ \\
\hline 6. O poder didático do aplicativo móvel será útil para mim. & $0(0 \%)$ & $0(0 \%)$ & $0(0 \%)$ & $3(75 \%)$ & $1(25 \%)$ \\
\hline \multicolumn{6}{|l|}{ Confiança } \\
\hline 7. O aplicativo móvel foi mais difíil de entender do que eu gostaria. & $1(25 \%)$ & $3(75 \%)$ & $0(0 \%)$ & $0(0 \%)$ & $0(0 \%)$ \\
\hline 8. O aplicativo móvel tinha tanta informação que foi dificil identificar e lembrar dos pontos importantes. & $0(0 \%)$ & $0(0 \%)$ & $2(50 \%)$ & $2(50 \%)$ & $0(0 \%)$ \\
\hline 9. O conteúdo do aplicativo móvel é tão abstrato que foi difícil manter a atenção nele. & $100 \%$ & $0(0 \%)$ & $0(0 \%)$ & $0(0 \%)$ & $0(0 \%)$ \\
\hline 10. As atividades do aplicativo móvel foram muito dificeis. & $100 \%$ & $0(0 \%)$ & $0(0 \%)$ & $0(0 \%)$ & $0(0 \%)$ \\
\hline $\begin{array}{l}\text { 11. Eu não consegui entender uma boa parcela do conteúdo e comportamento exposto no aplicativo } \\
\text { móvel. }\end{array}$ & $100 \%$ & $0(0 \%)$ & $0(0 \%)$ & $0(0 \%)$ & $0(0 \%)$ \\
\hline
\end{tabular}

Tabela 2. Distribuição dos juízes quanto ao nível de concordância com as afirmativas a respeito do parâmetro conhecimento feitas sobre o aplicativo avaliado. Belém, 2018

\begin{tabular}{|c|c|c|c|c|c|}
\hline \multirow[b]{2}{*}{ Aspectos e afirmativas } & \multicolumn{5}{|c|}{ Nível de concordância } \\
\hline & $\begin{array}{c}\text { Discordo } \\
\text { fortemente } \\
n(\%)\end{array}$ & $\begin{array}{c}\text { Discordo } \\
n(\%)\end{array}$ & $\begin{array}{c}\text { Neutro } \\
n(\%)\end{array}$ & $\begin{array}{c}\text { Concordo } \\
n(\%)\end{array}$ & $\begin{array}{c}\text { Concordo } \\
\text { fortemente } \\
n(\%)\end{array}$ \\
\hline \multicolumn{6}{|l|}{ Conhecimento } \\
\hline $\begin{array}{l}\text { 12. Depois do aplicativo móvel consigo lembrar-me de mais informaçôes relacionadas às manobras de } \\
\text { higiene brônquica apresentadas por ele. }\end{array}$ & $0(0 \%)$ & $0(0 \%)$ & $0(0 \%)$ & $3(75 \%)$ & $1(25 \%)$ \\
\hline \multicolumn{6}{|l|}{ Compreensão } \\
\hline 13. Depois do aplicativo móvel consigo compreender melhor as manobras de higiene brônquica. & $0(0 \%)$ & $0(0 \%)$ & $0(0 \%)$ & $4(100 \%)$ & $0(0 \%)$ \\
\hline \multicolumn{6}{|l|}{ Aplicação } \\
\hline 14. Depois de usar o aplicativo móvel sinto que consigo aplicar melhor o que aprendi. & $0(0 \%)$ & $0(0 \%)$ & $0(0 \%)$ & $3(75 \%)$ & $1(25 \%)$ \\
\hline
\end{tabular}


Os juízes que avaliaram o aplicativo móvel caracterizaram-se por profissionais fisioterapeutas que atuam como docentes em cursos de fisioterapia, com tempo de experiência profissional entre $10 \mathrm{a}$ 15 anos e com formação acadêmica, na sua maioria composta por mestre. Porém no estudo de Galvão e Püschel ${ }^{13}$ encontramos achados diferentes, os autores afirmam que a maioria dos avaliadores eram especialistas e possuíam mais de cinco anos de atividade docente. $E$ também, no estudo de Tibes ${ }^{12}$ pelo menos $37 \%$ dos avaliadores apresentam mestrado ou doutorado na área e $50 \%$ eram especialistas.

Entre as características dos juízes, pode-se destacar, que todos são docentes do curso de fisioterapia, em atividade a 15 anos e que estimula a capacitação dos seus professores. Acreditamos que isso possa ter influenciado no tempo de docência e na titulação dos avaliadores. No que se refere ao nível de motivação, os juízes foram $50 \%$ (02/04) concordantes com a afirmativa que abordava o aspecto 'Atenção' e os demais mantiveram-se 'neutros' a esse respeito.

Consideramos esses achados pois, as filmagens foram realizadas e editadas pela equipe profissional da produtora e o aplicativo móvel, em parceria com especialistas de computação.

Corroborando com os achados citados, os autores Galvão e Püschel ${ }^{13}$ afirmam em seu estudo, que à interface do ambiente, design das telas, organização dos conteúdos, tamanho de letras e cores adotadas, obtiveram classificação ótima pela maioria dos especialistas.

No tocante ao aspecto 'Relevância', todos os juízes concordaram 'fortemente' com as afirmativas que avaliaram o conteúdo contido no aplicativo quanto a sua clareza, relevância para o ensino e relação com o que fizeram ou pensaram em termos de ensino docente. Sobre a motivação para 'aprender mais sobre o assunto abordado', 100\% (04/04) dos avaliadores foram, no mínimo, concordantes com esse quesito, sendo que $75 \%$ (03/04) deles concordaram 'fortemente'; 75\% (03/04) dos juízes concordaram que o "poder didático do aplicativo" Ihes é útil e os demais foram 'fortemente' concordantes com essa afirmação.

Galvão e Püschel ${ }^{13}$ ressaltam em sua pesquisa, que os itens relevância do tema e coerên cia dos conteúdos foram avaliados como ótimos por todos os especialistas. Confirmando esses achados encontrou-se no estudo de Tibes $^{12}$ que $100 \%$ dos fisioterapeutas entrevistados, assinalaram que os assuntos presentes no aplicativo se relacionam com o que foi visto no período de graduação em Fisioterapia e/ou do que se utiliza na prática da Fisioterapia.

Na seleção e elaboração do conteúdo tivemos a preocupação em disponibilizar os conceitos de forma clara, com uso de vocabulário simples e informações suficientes, por meio de textos curtos para evitar a ambiguidade e permitir a transmissão e captação das mensagens. Os textos devem permitir a fácil leitura e rapidamente promover e obter os resultados esperados de aprendizagem. A escolha e apresentação do conteúdo deve levar em consideração a sua capacidade em acionar os conhecimentos prévios dos estudantes ${ }^{14}$.

Na avaliação do impacto do aplicativo na aprendizagem dos alunos, foram avaliados os aspectos 'conhecimento', 'compreensão' e 'aplicação', baseados nos níveis homônimos da Taxonomia de Bloom ${ }^{15}$. Quanto ao 'Conhecimento', 75\% (03/04) dos juízes foram concordantes e $25 \%(01 / 04)$ 'fortemente' concordantes com o fato de que o aplicativo auxiliou na lembrança de "mais informações relacionadas às manobras de higiene brônquica" apresentadas e as mesmas proporções de respostas foram observadas na avaliação do aspecto 'Aplicação'. No que diz respeito à 'Compreensão', os avaliadores foram unânimes em 'concordar' que o aplicativo auxiliou na melhor compreensão das manobras em questão. 
No ponto que o aplicativo auxilia na lembrança de informações relacionadas às manobras de higiene brônquica, foi encontrado achados semelhantes no estudo de Gonçalves e Melo ${ }^{16}$ identificou-se que a maioria dos entrevistados consideram que o aplicativo 'FISIOSPITAL' apresentado na forma de dispositivo móvel facilitou o entendimento e existe compatibilidade entre os assuntos abordados e a prática da Fisioterapia. Entretanto, alguns entrevistados referiram que o aplicativo poderia ser apresentado de outra forma, porém percebeu que, usando o aplicativo, houve agregação de valor aos assuntos nele abordados.

Gonçalves e Melo ${ }^{16}$ ainda pergunta sobre a primeira impressão ao tentar utilizar o aplicativo 'FISIOSPITAL', observou-se a maior parte da amostra, referindo que não havia complicação para empregar o aplicativo e logo foi entendido como utilizá-lo de várias maneiras. Consideramos a avaliação do aplicativo móvel quando realizada por um especialista no assunto, é de grande valia. Assim podemos conhecer os problemas encontrados e melhorá-los para uma melhor usabilidade. Pactuando com esse pensamento Tibes $^{12}$ declara, que a avaliação dos especialistas foi de fundamental importância para adequação e melhoria do seu aplicativo.

\section{Avaliação do aplicativo móvel pelos alunos}

A qualidade do aplicativo foi avaliada pelos alunos do curso de fisioterapia por meio de perguntas que abordavam diferentes características, cujas respostas são apresentadas a seguir. Enquanto "instrumento de aprendizagem" e "ferramenta de estudo", a maioria significativa dos alunos considerou o aplicativo avaliado como 'excelente' nesses quesitos $(60 \%, 24 / 40$; $p<0,0001$ e 70\%, 28/40; $p<0,0001$, respectivamente) e, de modo semelhante, a maioria avaliou como 'excelente' sua facilidade de manuseio $(60 \%, 24 / 40$; $\mathrm{p}<0,0001)$. Achados semelhantes foram encontrados no estudo de Galvão e Püschel13 afirmando que os aspectos educacionais do seu aplicativo, foram avaliados por fisioterapeutas como ótimo e satisfatório.

Segundo Silva et al. ${ }^{10}$ em seu estudo, o uso de um recurso multimídia on-line teve um impacto positivo no aprendizado de estudantes de fisioterapia respiratória, área na qual os recursos instrumentais e manuais são frequentemente utilizados e podem ser explorados no contexto deste tipo de tecnologia.
Consideramos que a utilização de vídeos no aplicativo móvel, tenha influenciado na avaliação positiva no aspecto aprendizagem, pois a qualidade das imagens, que se apresentam em movimento, faz com que a mensagem a ser compartilhada se aproxime ainda mais da realidade. Segundo Vieira ${ }^{17}$ os vídeos podem dar contexto e adequação aos ambientes de aprendizagem, podendo ainda ser uma ferramenta de raciocínio por permitir ao espectador selecionar o conteúdo ao qual quer ter conhecimento, parar, avançar, voltar, anotar, estabelecendo relações do vídeo com outras fontes de informação.

O estudo de Gonçalves e Melo ${ }^{16}$ descreve que nas categorias analisadas, referentes à interface, usabilidade, praticidade e aplicabilidade, obteve-se $100 \%$ dos entrevistados concordando totalmente que o aplicativo é fácil de ser entendido e utilizado; que facilitou a prática clínica, e que o utilizaria constantemente.

Acreditamos que o uso do aplicativo móvel possa ter incentivado aprendizagem, tornando-a menos formal através dessa ferramenta complementar, que enriquece e melhora o sistema de ensino.

Como "instrumento de auxílio para entender as manobras" apresentadas, a maioria significativa dos alunos avaliou que o aplicativo é, no mínimo, 'muito bom' (60\%, 24/40), sendo 'excelente' para 30\% (12/40) dos respondentes $(p<0,0001)$. Na avaliação da utilidade do aplicativo na promoção de "dados concretos para o ensino das manobras de higiene brônquica", houve igual proporção de alunos que o consideraram 'boa' e 'excelente' ferramenta (40\%, 16/40; $p<0,0001)$, e como auxiliar na "prática da fisioterapia", a maioria $(50 \%, 20 / 40)$ dos alunos o julgou 'excelente' para esse fim e $40 \%$ (16/40) o considerou como 'muito' boa ferramenta para esse propósito, sendo significativas essas proporções $(p<0,0001)$.

Entendemos esses achados, devido a literatura sobre as manobras de higiene brônquica, não descreverem de maneira satisfatória suas técnicas, talvez por se tratarem de técnicas exclusivamente manuais e exigir muito mais que experiência prática de quem se aplica do que de sua própria fundamentação cientifica. As técnicas acabam sendo adaptadas de acordo com a preferência individual dos terapeutas, muitas vezes descaracterizando a manobra originalmente descrita ${ }^{11}$. 
Segundo Gonçalves e Melo16 em seu estudo, quando avaliou o proveito do aplicativo, observou-se uma heterogeneidade nas respostas dos participantes da pesquisa, correspondendo a 46,6\% da amostra, afirmaram que o aplicativo é proveitoso, porém precisa de ajustes para ajudar o entendimento dos fisioterapeutas. Já $26,7 \%$ referiram que o aplicativo da pesquisa não precisa ser melhorado, mas que o usuário precisa de mais tempo de uso para aproveitar melhor os assuntos abordados. Na mesma proporção, responderam que o aplicativo não precisa ser melhorado, pois já está pronto para ser utilizado pelos fisioterapeutas.

A figura 1 ilustra a distribuição dos alunos quanto a avaliação geral do aplicativo móvel contendo manobras de higiene brônquica. Observou-se proporção igual de alunos que o considerou 'bom' e 'excelente' (40\%, 16/40), sendo que estes representavam a maioria significativa dos respondentes $(p<0,0001)$.

Figura 1. Distribuição dos alunos quanto a opinião geral a respeito do aplicativo móvel avaliado. Belém, 2018

\section{Pergunta 7. Após o uso do aplicativo móvel de higiene brônquica, como você o considera?}

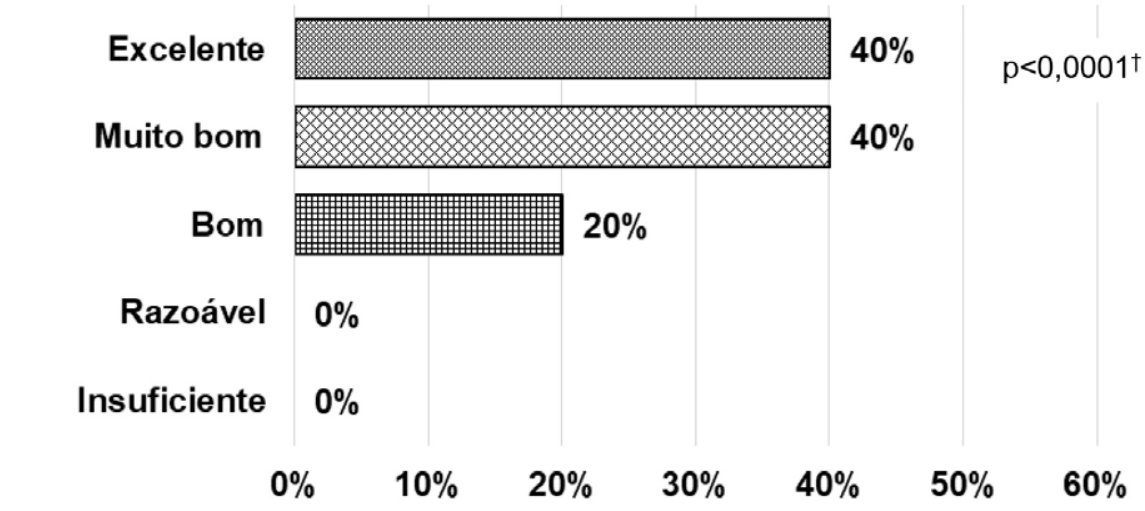

Estatisticamente significativo (Qui-Quadrado de aderência).

Fonte: Questionário da pesquisa, 2018.

Confirmando esses achados, Gonçalves e Melo ${ }^{16}$ afirmou que o uso de aplicativo por fisioterapeutas mostrou-se de grande valor na contribuição e na facilitação de assuntos direcionados à fisioterapia respiratória quando associados à prática diária da fisioterapia.

Durante a coleta de dados, alguns alunos fizeram algumas sugestões. Sugeriram o acréscimo de faixas de áudio, ou elaboração de vídeo que associa imagem e som, como recursos complementares.

O uso de novas tecnologias também pode apresenta barreiras que parecem estar mais relacionadas a problemas técnicos e fatores sociais e de aptidão do aluno. Segundo Silva et al. ${ }^{10}$ as maiores barreiras relatadas por alunos para o uso das novas tecnologias: maior susceptibilidade a colapso, os alunos se consideram incapazes de trabaIhar com computador e/ou acreditam que a experiência mediada por computador não substitui a aula presencial.

Obviamente, a tecnologia não ensina sozinha, alunos não aprendem de meios tecnológicos, mas de instrutores competentes que devem ter sido treinados a se comunicar através desses meios. Acreditamos que o APP desenvolvido possa auxiliar no ensino e que este modelo possa ser aplicado ao ensino de diversas outras disciplinas, uma vez que estudos realizados em outras áreas da saúde já vêm sugerindo que o uso de novas tecnologias pode facilitar o aprendizado dos alunos. 
Pensando no enriquecimento do processo ensinoaprendizagem e na necessidade de acompanhar as evoluções tecnológicas, enfatiza-se a importância de desenvolver ferramentas que busquem uma transformação significativa na educação e que representem mais um meio de atingir os objetivos educacionais e melhorar a qualidade do ensino ${ }^{13}$.

Nesse estudo encontramos uma pequena limitação a respeito da velocidade de transmissão dos dados dos smartphone, sendo sugerido pelos alunos, a utilização do aplicativo móvel sem necessidade de internet.

\section{Conclusão}

Foi desenvolvido um aplicativo multimídia, que pode oferecer ao estudante a oportunidade de melhorar a relação da teoria com a prática, de estabelecer uma correlação entre o que aprende e as situações reais, tornando a aprendizagem mais significati $\neg v a$, mais enriquecedora.

O aplicativo ainda passou por avaliação de qualidade técnica e funcional, realizada por juízes, no qual eles descreveram a concordância com uma série de afirmações que abordavam os parâmetros nível de motivação e impacto na aprendizagem produzidos pelo aplicativo móvel. Após a análise das respostas, concluirmos, em sua totalidade, a satisfação dos juízes, salientando que o aplicativo é prático, de fácil entendimento e pode auxiliar na aprendizagem.

$\mathrm{Na}$ avalição das opiniões geradas pelos alunos, quanto a utilização do aplicativo móvel proposto para o processo de ensino-aprendizagem, nos aspectos, instrumento de aprendizagem, ferramenta de estudo, facilidade de manuseio, na promoção de dados concretos para o ensino das manobras de higiene brônquica, auxiliar na prática da fisioterapia, a maioria significativa dos alunos considerou o aplicativo avaliado como excelente. Ainda na opinião dos alunos, analisando o aplicativo como instrumento de auxílio para entender as manobras apresentadas, sua maioria significativa avaliou que o aplicativo é, no mínimo, muito bom. Na análise geral do aplicativo móvel contendo manobras de higiene brônquica. Observou-se proporção igual de alunos que o considerou bom e excelente, evidenciando a concordância entre os diferentes avaliadores.

Compreendemos que a inserção de tecnologias para o processo de educação é executável e se comporta como um complemento, podendo ser inserido como educação permanente aos graduandos e profissionais de fisioterapia.

\section{Contribuição dos autores}

Santos RC idealizou o projeto, fez coleta de dados, pesquisas bibliográficas necessárias ao trabalho e redação. Gomes PAF idealizou, orientou metodologicamente e contribuiu com resultado, discussão e redação final.

\section{Conflitos de interesses}

Nenhum conflito financeiro, legal ou político envolvendo terceiros (governo, empresas e fundações privadas, etc.) foi declarado para nenhum aspecto do trabalho submetido (incluindo mas não limitando-se a subvenções e financiamentos, participação em conselho consultivo, desenho de estudo, preparação de manuscrito, análise estatística, etc.).

\section{Referências}

1. Nogueira, MI. As mudanças na educação médica brasileira em perspectiva: reflexões sobre a emergência de um novo estilo de pensamento. Rev Bras Educ Med. 2009;33(2):262-270. doi: 10.1590/S0100-55022009000200014

2. Chiesa AM, Nascimento DDG, Braccialli LAD, Oliveira MAC, Ciampone MHT. A formação de profissionais da saúde: aprendizagem significativa à luz da promoção da saúde. Revista Cogitare Enferm. 2007;12(2):236-240. doi: 10.5380/ce.v12i2.9829

3. Arruda FT, Danek A, Abrão KC, Quilici AP. Elaboração de vídeos médicos educacionais para treinamento de habilidades de estudantes do curso de medicina. Rev Bras Educ Med. 2012;36(3):431-435. doi: 10.1590/S0100-55022012000500019 
4. Moraes LF, Fioco EM, Silva GP, Ferreira B, Verri ED. O uso dos mini vídeos - aulas como ferramenta de aprendizagem da interação do conhecimento básico com o clínico da disciplina de anatomia humana do centro universitário Claretiano de Batatais. Revista da Liga Acadêmica. 2012;2(2):209-215.

5. Guerreiro JRG, Battini O. Novas tecnologias na educação básica: Desafios ou possibilidades? In: JORNADA DE DIDÁTICAS: DESAFIOS PARA A DOCÊNCIA. 3, 2014, Londrina. Anais da III Jornada de didáticas: Desafios para a docência. Universidade Estadual de Londrina, 2014. P. 297-307.

6. Vieira, MM. Educação e novas tecnologias: o papel do professor nesse cenário de inovações. Revista Espaço Acadêmico. 2012;11(129):95-102.

7. Itakussu EY, Fujisawa DS, Franco PPR, Schultz AR, Anami EHT, Kuwahara RM, Trelha CS. Elaboração de vídeo educativo sobre uso da malha compressiva após queimaduras. Revista Brasileira de Queimaduras. 2014;13(14):236-239.

8. Pastor Junior AA, Rezende LAC, Bastos WG. Recepção audiovisual na educação médica: Leitura de um vídeo educativo de psicologia médica por estudantes de medicina. Rev Bras Educ Med. 2012;36(4):516-523.

9. Mayer RE. The promise of multimedia learning: using the same instructional design methods across diferente media. Learnign and Instruction. 2003;13(20):125-139. doi: 10.1016/S09594752(02)00016-6

10. Silva CCBM, Toledo SLP, Silveira PSP, Carvalho CRF. Evaluation of a multimedia online tool for teaching bronchial hygiene to physical therapy students. Revista Brasileira de Fisioterapia. Rev Bras Fisioter. 2012;16(1):68-73.

11. Sarmento, GJV. Fisioterapia Respiratória no Paciente Crítico:

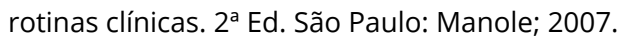

12. Tibes CMS. Aplicativo móvel para prevenção e classificação de úlceras por pressão. [dissertação]. São Carlos: Universidade Federal de São Carlos; 2014.

13. Galvão ECF, Püschel VAA. Aplicativo multimídia em plataforma móvel para o ensino da mensuração da pressão venosa central. Revista da Escola de Enfermagem da USP. 2012;46(Esp):107-15. doi: $10.1590 / S 0080-62342012000700016$

14. Jucá SCS. A relevância dos softwares educativos na educação profissional. Ciências \& Cognição. 2006;8. 22-28.
15. Savi R, Christiane, von Wangenheim CG, Ulbricht V, Vanzin T. Proposta de um Modelo de Avaliação de Jogos Educacionais, Novas Tecnologias na Educação, CINTED-UFRGS. 2010;8(3):1-12.

16. Gonçalves GC, Melo AM. Avaliação de um aplicativo tecnológico por fisioterapeutas da Atenção Primária em Saúde. RE SAÚD DIGI TEC EDU.2017;2(1):03-20.

17. Vieira FMS. A utilização das novas tecnologias na educação numa perspectiva construtivista. 2009. 